\title{
Drawing From Social Media to Inspire Increasingly Playful and Social Drone Futures
}

\author{
Alexandra Pometko \\ Social Emotional Technology Lab, \\ UC Santa Cruz \\ apometko@gmail.com
}

\author{
Ella Dagan \\ Social Emotional Technology Lab, \\ UC Santa Cruz \\ ella@ucsc.edu
}

\author{
Ferran Altarriba Bertran \\ Social Emotional Technology Lab, \\ UC Santa Cruz \\ ferranaltarriba@gmail.com
}

\author{
Katherine Isbister \\ Social Emotional Technology Lab, \\ UC Santa Cruz \\ kisbiste@ucsc.edu
}

\begin{abstract}
In this pictorial, we explore the potential of social media to help inspire ideas for future drone design applications that support playful and social experiences. Drawing from a Situated Play Design approach [3], we turn to social media posts to identify recurring playful and social instances of drone use in social settings. We present the results of collecting 143 posts found on Instagram, TikTok, and YouTube, from which we identified a non-exhaustive list of drone-based play potentials, i.e. existing ways in which people already appropriate drones to playfully augment social situations. We present these play potentials as potentially valuable and inherently situated intermediate-level knowledge with generative power. We argue they might inspire the design of future drone technologies and experiences in Human-Drone Interaction (HDI), in directions that increasingly respond to people's desires for play and social connection.
\end{abstract}

\section{Authors Keywords}

Human-Drone Interaction; Playfulness; Play potentials; Situated Play Design; Social media.

Permission to make digital or hard copies of part or all of this work for personal or classroom use is granted without fee provided that copies are not made or distributed for profit or commercial advantage and that copies bear this notice an the full citation on the first page. Copyrights for third-party components of this work must be honored. For all other uses, contact the Owner/Author.

DIS '21, June 28-July 2, 2021, Virtual Event, USA (C) 2021 Copyright is held by the owner/author(s). ACM ISBN 978-1-4503-8476-6/21/06. https://doi.org/10.1145/3461778.3462020

\section{CSS Concepts}

- Human-centered computing Interaction design

\section{INTRODUCTION}

The design and research space of Human-Drone Interaction (HDI) is still relatively new, and existing work is mostly concerned with technical and utilitarian research agendas. At a societal level, we see something similar: drones are still at an incipient stage of their integration into people's lives, and recurrent use cases are often productivity-focused. For example, drones are used to assist in search and rescue missions [13], to sanitize surfaces during the COVID-19 pandemic [19], or to help with surveillance [21] and related uses in the military [16], among others. Here we argue that, just as many other emerging technologies, drones have the potential to serve alternative, less utilitarian goals.

Exploring how drones might afford experiences that are not necessarily productive in a material sense is not a dominant research agenda in Human-Computer Interaction. However, there are some works that explore alternative avenues for HDI. For example, in an effort to create more natural and engaging HDI, work has been done to provide drones with emotion recognition capabilities, as well to encode emotions in drones that can be signaled to people by using the robot's flight patterns $[6,11]$ (e.g. making a drone fly very fast in a non-linear pattern to make it look anxious). Other works explore how drones could be used to augment physical activities, like a drone to practice movements inspired from Tai-Chi [15], a telecommunication drone for athletes' encouragement [20], a drone that projects playgrounds for street games [14], or a drone used as a ball to change the physical properties of a traditional ball to affect the gameplay [17].

Here we add to this body of work by exploring how drones might support experiences of social and playful interaction. Unlike the previous works that present the development of a specific design and its evaluation, we take a step back and explore the kinds of playful and social things people enjoy doing with drones in naturalistic settings, with the objective of inspiring future designs. Play is "an essential way of engaging with and learning about our world and ourselves" [8] and creates opportunities for well-being, creativity, self-expression, learning, and meaningful social interaction [3]. As such, it can enrich our interactions with technology and contribute to nurturing a number of societal values that, though they might not directly be materially productive, are directly consequential to human flourishing [1]. We see the overall lack of playful and non-utilitarian research as a missed opportunity, and we argue that playful design could be a way to explore alternative avenues for HDI.

To explore this gap in ways that are contextually meaningful, we take a Situated Play Design approach [1]. We examine how people already use drones "in the wild", building on the social and playful practices people are already doing with them. That is a particularly interesting approach in the design space of drones. Insofar as they are a growing market [12] and an emergent product, the 
role of drones in society is still under construction. As a result, drone consumers are continually inventing and discovering new uses for them, both on the utilitarian and on the social and entertainment fronts. Arguably, those emergent and consumer-driven ways of using drones are highly meaningful to people, and as such, they can be a valuable inspiration for drone technology design. Here we argue that consumer-driven and spontaneous ways of using drones could and should be considered more in HDI, both in academia and in commercial design.

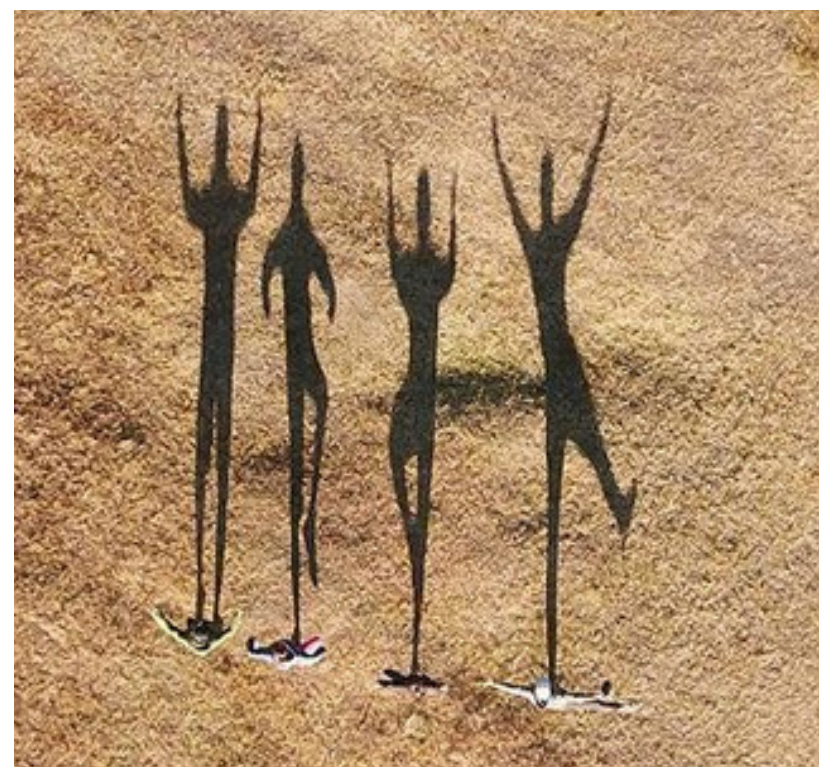

An example of a spontaneous playful appropriation: Adopting the drone's perspective to play with shadows in the outdoors. Credits: debetchris, Instagram

Our explorations of existing playful and social practices with drones allowed us to surface a series of play potentials [3], i.e. existing and contextually-meaningful playful practices people do with drones in social settings. To identify those play potentials, we took inspiration from [2] and explored people's accounts of their drone practices on social media. We created a collection of
143 posts, from which we identified six interesting play potentials that build on people's existing playful interaction with drones in social settings. The play potentials we present in this pictorial (which we frame as an annotated portfolio [7]), highlight the design qualities, affordances, and interaction mechanisms we found interesting in people's existing uses of drones in social settings. We believe that they hold generative power, as they could be inspirational for designers and help to spark ideas for increasingly playful and social drone-based experiences. The play potentials include accompanying use examples.

These examples of actionable interaction mechanisms and modalities could be built upon (for example in brainstorming sessions), and could inspire ideas that might not have surfaced through other formal lab studies (like the situation represented on the left in "An example of a spontaneous playful appropration"). In the discussion, we present how this work inspired our own generation of playful drone application ideas, and how other designers can do the same by using this pictorial or by doing their own social media exploration.

\section{METHOD}

To explore how people already make playful and social uses of drones, we turned to the Situated Play Design (SPD) [3] methodology, aimed at sourcing design inspiration in people's existing, ordinary practices. SPD builds on the idea of "uncovering existing manifestations of contextual playful engagement", which the authors call play potentials, and using those to inspire increasingly contextually meaningful playful experiences and technologies.

In this study, we were inspired by recent work exploring how to chase play potentials through social media with inspirational purposes [2]. However, unlike in [2] where the researchers themselves created Instagram posts to document play potentials they encountered in the wild, we used social media to explore and curate the playful uses of drones that had already been captured by other people, without our intervention. This approach allowed us to access uses of drones consumers themselves found fun. Further, it also worked well in the circumstances surrounding the timing of our study: it was an effective strategy for accessing a wide range of practices with drones around the world when the COVID-19 pandemic imposed strict limitations for accessing stakeholders for in-person interviews and workshops.

We chose to use three social media platforms, Instagram, TikTok, and YouTube, for four reasons: i) the media posted on these platforms is highly visual, which would allow us to better understand the interactions between the drones and people; ii) we knew from our personal experience that high amounts of drone content could be found on these platforms; iii) these platforms were amongst the most popular social media platforms during the time of this study [4]; iv) each of these platforms offers different affordances that might influence the type of content people post and the manner in which humandrone interaction is presented, and we thought could potentially lead to different types of play potentials related to each platform. By using three different social media platforms we hoped to increase the diversity of the content that we explored.

\section{Privacy considerations}

Before gathering user data from these platforms, we needed to address ethical and legal considerations, to determine if we could use social media posts in our research project. We consulted our US-based IRB Office who reassured us that in the US and EU, if the post was uploaded publically (e.g., on an Instagram profile that is not private, for which other users of the platforms can access the content without needing to request specific permission from the owner to view the content on their profile), it could be used freely in our research without asking for the owner's consent or consent from any other third party that might appear in the post. However, in the case of private posts, consent must be obtained from the owner and any third party according to their country's regulations. In this study, we only gathered public posts, and we credit and acknowledge the authors' effort in posting novel uses of drones. 


\section{Method: How we identified play potentials from social media posts}

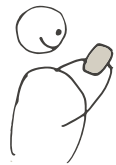

\section{Step 1: Collecting posts}

One of the four design researchers of the study began their exploration by collecting relevant posts from Instagram, TikTok, and YouTube using their own private accounts. To do so, they used a range of search keywords related to play and playfulness, such as combinations of "drone" with "fun", "play", "social", and "friend" on each platform's search feature. The researcher browsed many posts associated with these keywords (the total number is unknown because social media platforms do not allow easy counting and monitoring of viewed posts) and selected a small fraction of those, coming up to a total of 143 posts based on their personal appreciation of whether the post featured a playful, social, or non-utilitarian use of drones. They stopped collecting posts when the ones suggested by the platforms started getting repetitive or when the end of a specific keyword search results was reached.

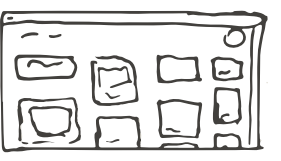

\section{Step 2: Organizing the} posts in a Pinterest board

Presenting these posts to the rest of the team was challenging as they were all saved on three different platforms. To overcome the challenge of presenting them as a collection, all posts were added to a Pinterest board. This allowed us to centralize the content and visualize a preview image for each post, while still maintaining the URL links to the original posts. First, posts were clustered by the first researcher in groups using inductive thematic analysis, which resulted in a series of emerging themes (e.g., a theme for posts featuring drones and animals, another for drones in cooking scenarios, etc.). These themes helped us identify recurrent playful and social interaction mechanisms. A total of 24 drone-based interaction themes were instantiated and supported by a series of social media posts in our collection. The groups were then reviewed and validated by the 3 other researchers.

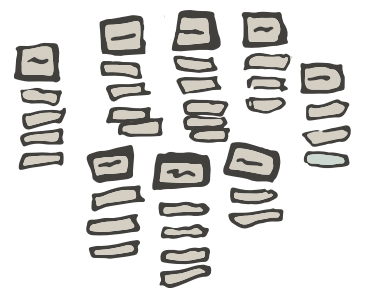

Step 3: Clustering similar groups into broader categories

As we found many similarities in the ways drones were used in the 24 initial themes, the first researcher did a second round of clustering through a card sorting exercise [22] using Trello (a web application that allows dragand-dropping of items). That allowed us to iteratively narrow down our initial list of themes into a set of fewer and broader categories.

\section{Step 4: Reviewing and pruning}

From the initial set of themes, the four researchers narrowed down to a final list of 6 categories - that is, 6 recurrent forms of playful and social engagement with drones that we found in our collection of social media posts.

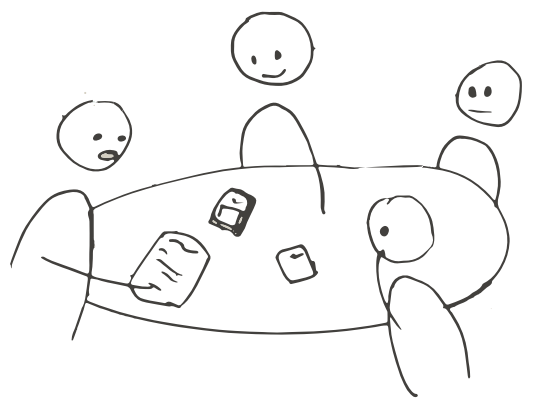

We refined that final set of play potentials by eliminating repetition and by removing themes that had fewer instances in our dataset. For example, we removed a theme about drone customizations (e.g. adding clothes or physical attributes to the drone) because we found it had less than 10 instances and it strongly overlapped with the "Animate" theme (which was about ways in which people make drones 'come to life' by attributing them human or animal qualities, see p. 6). Our final selection of drone-based play potentials reflects what we thought was most relevant, inspiring, and actionable to generate new ideas based on our knowledge and specialization in playful, social, and embodied interaction design.

\section{Results}

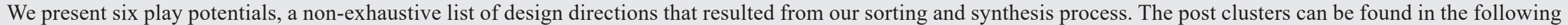
Pinterest board: https://bit.ly/3eka6bi. Our aim with the play potentials is to propose an inspirational point of departure for designers of future HDI works.

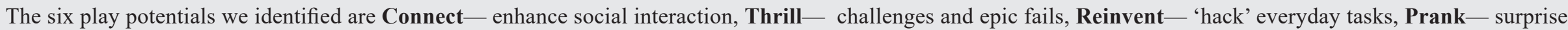
and delight, Animate - make drones 'come to life', and Create - aerial artistic medium.

Here we present them as generative design directions illustrated with selected social media posts that inspired them. 


\section{Drone-based play potentials and guiding inspirational questions}

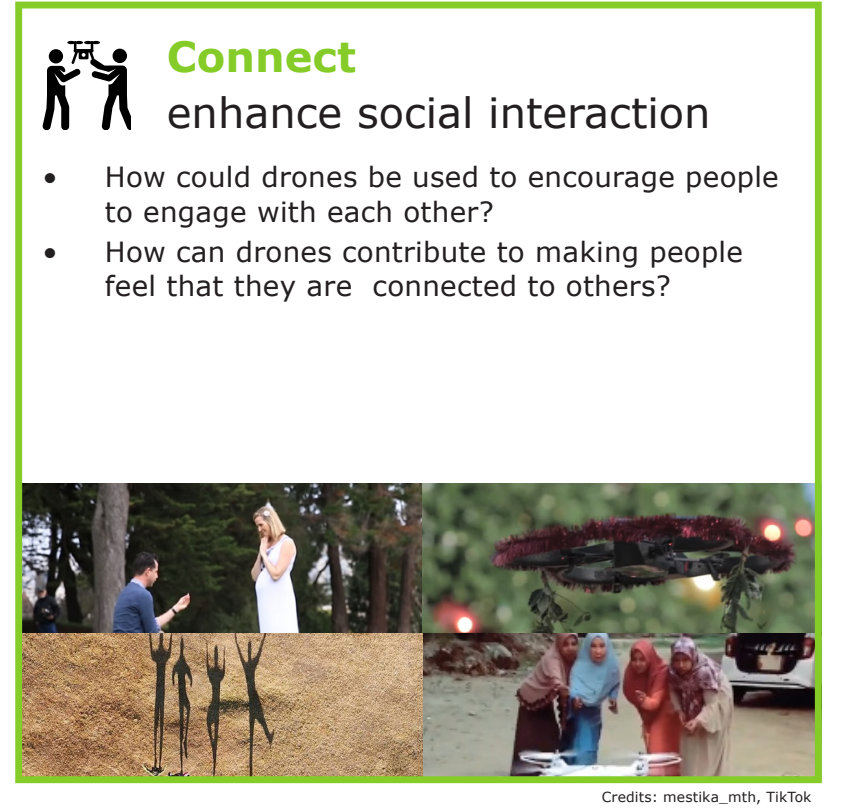

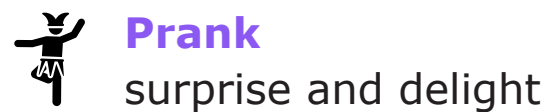

- How could we use drones to create surprising moments or delight people?

- What are original types of pranks we can only craft with drones?

- Could there be something fun about framing the drone as a character with its own personality?

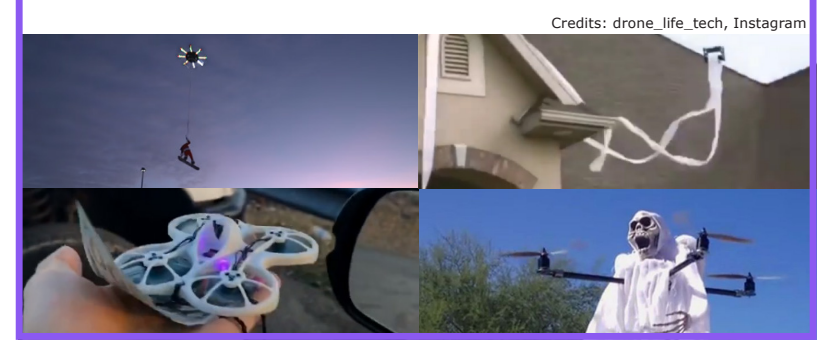

(3) Thrill

challenges and epic fails

- What role could drones play in physical challenges (e.g. target, protagonist, facilitator...)?

- What could be fun challenges involving drones and how can they be exciting?

- How can drones lead to surprising, fun, and performative 'failures'?

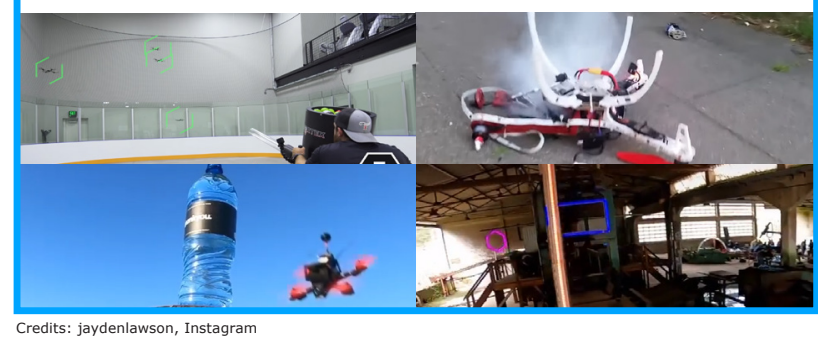

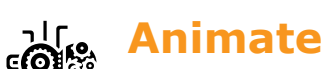 \\ make drones 'come to life'}

- How could people turn their drones into "living things"?

- How could those "living things" participate in a social setting (e.g. friend, referee, part of a living ecosystem...)?

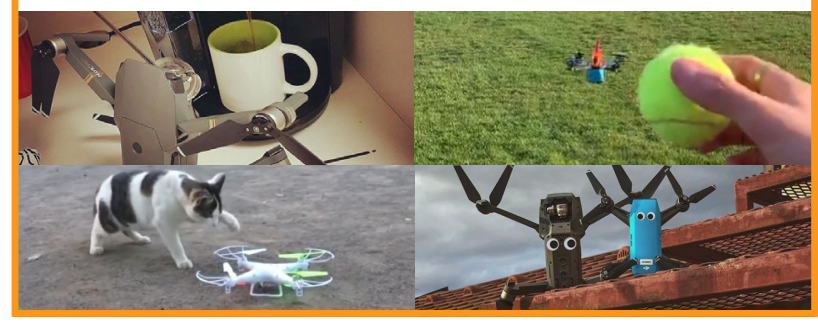

Icons by Freepik and Smashicons on flaticon.com

\section{证 Reinvent}

望

- How could drones enable free-form experimentations?

- How could drones be designed so that they are customizable and appropriable?

- In which ordinary human tasks might it be fun to use drones?

- How could we design for drone interactions that are both fun for users and bystanders?
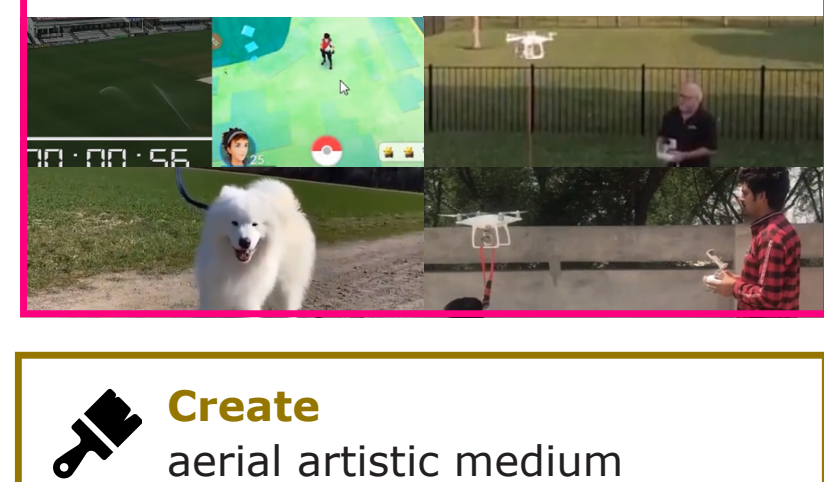

Create

aerial artistic medium

- How could drones contribute to enriching existing practices?

- How could drones add meaning to an artistic practice and the resulting artwork?

- How could we use drones in out of the ordinary ways to create art?

- How could drones enable new artistic languages?

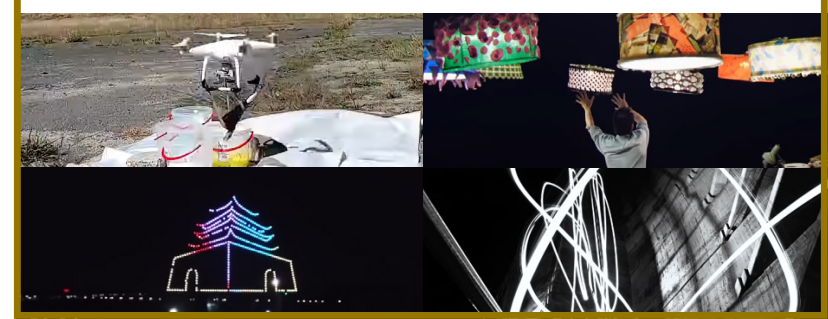




\section{DISCUSSION}

\section{From Drone-Based Play Potentials to Design}

Our approach is, in part, motivated by Gobeli and Brown, who found that most commercial products rely mainly on incremental innovation [10], i.e. products building upon existing technology and user habits, while Norman and Verganti highlighted the strengths of HumanCentered Design for incremental innovation as well as for user-driven innovations in meaning [18]. Because the drone-based play potentials are grounded in people's existing creative uses of drones in social settings, we argue that they can serve as inspiring design directions to help shape future HDI designs-directions that we argue could be increasingly contextually sensible. Here, a relevant question arises: how can these play potentials be used to effectively support the design process of nonutilitarian HDI design? We see the potential for using them in different design stages:

First, during the phase of contextual research, our contribution might provide designers with a starting point for how to begin their inquiries about people's drone uses and preferences. Our list of play potentials and design trajectories can be further developed with new data from methods like interviews, surveys, and focus groups with expert and non-expert drone users.

Second, during the early ideation phases, the presented play potentials might provide designers and other stakeholders a sense of existing uses. The play potentials can also be used as fundamental elements for brainstorming exercises, where ideas can be created within specific design trajectories, using the documented drone-interaction mechanisms and play potentials as building blocks. It may support coming up with more original, informed, and contextually meaningful ideas.

We used the play potentials in our ideation stage to produce a catalog of speculative social drone technologies ${ }^{1}$. We used the Crazy 8-like [25] brainstorming method to rapidly come up with about 3-4 ideas for each design trajectory, dedicating one minute

1 Link to the catalog: https://bit.ly/3sFH4Ip to each, which led to the creation of 35 ideas in one session. Then, we narrowed down and polished the set of ideas to a final list of 10 technology concepts that we formatted into a speculative drone design catalog that will be used to engage with stakeholders in a subsequent co-design phase.

Third, during the concept development and prototyping phases, the play potentials which are illustrated by the collection of posts and the guiding questions can help designers concretize their design ideas. After producing early concepts, the example social media posts can be used as inspiration for how to materialize the envisioned drone application more specifically. For example, when social media users customized their drones to paint, how did they attach the paintbrush to the drone? How did they dip the brush into paint? Was it successful? What other strategies could be used?

\section{Limitations}

Though our approach allowed us to source social and playful drone inspiration in people's diverse practices around the world, basing our study on social media content also brought about limitations. First, the posts we viewed were the result of using specific social media platforms, reflecting content created by the people who most commonly use them. This means that the content creators' demographic could be skewed towards specific nationality. For instance, China is one of the countries with the most amateur drone users with a market estimated to be $\$ 10.9$ billion by 2025 [24]: we probably would have uncovered more drone applications if we had surveyed WeChat rather than the social media platforms we have (we chose platforms that were most familiar to us as researchers living in the United States). As part of a research through design approach [9], we do not seek to paint an exhaustive picture of all Human-Drone interaction modalities that exist in the wild. Rather, as Gaver suggests: "Design, and research through design, is generative. Rather than making statements about what is, design is concerned with creating what might be, and moreover, in Zimmerman et al.'s formulation, on making the "right thing" [9]. Our work aims to inspire the generation of ideas rather than provide an accurate representation of the world. Gathering more data could certainly uncover new types of play potentials, but in our case, the limited amount of data (143 posts) collected allowed us to engage and consider each post qualitatively. Also, these play potentials are not completely disjoint, as some posts can sometimes overlap two play potentials (e.g. a prank creating an opportunity to connect). However, their core interaction mechanisms are sufficiently distinct to make them unique.

Another limitation is that the content we encountered was presented to us by each social media platform's recommendation algorithm that suggests content based on the user's search and interest history. To collect the posts, the researcher used their personal Instagram and YouTube accounts that were more than three years old (while creating a brand new account of TikTok for the objectives of this research). There is no way to know which posts were omitted in that manner that might have been valuable for this research, or which ones were put forward due to the researcher's other interests explored in the platforms previously.

One final limitation is that the drone uses we discovered must all be looked at through the lens of social media which adds affordances that are not present when drones are used regardless of being documented and posted For example, its performativity affordances such as camera recording are being leveraged for creating content to later post on social media. Some of these play potentials can maybe only exist within that realm, which must be considered by designers. Moreover, it also implies that many other playful drone uses have probably gone undocumented. The play potentials we found are also limited to the interactions possible with the current affordances and limitations of drone technology. Observing future technology improvements or even taking a look at science-fiction work could help us surface other types of play potentials. This suggests that more research needs to be done to enable Situated Play Design in HDI. 


\section{CONCLUSION}

In this pictorial, we presented six play potentials that highlight ways in which people appropriate drones in social settings. We discovered them by collecting and thematically organizing 143 social media posts (from Instagram, TikTok, and YouTube). Each play potential is concretized and instantiated by examples of posts in our collection. We present these play potentials as six design trajectories that can inspire the design of increasingly playful and social interactions with, around, and through drones. For each play potential, we offer a set of guiding questions designers and researchers can use to inspire their practice. We believe that our work can give rise to future Human-Drone Interaction designs that enable types of social experiences that have not been explored before.

\section{ACKNOWLEDGMENTS}

We thank all the authors of the posts featured in this pictorial for their publicly-made showcases of original drone uses. We also want to thank our reviewers for their valuable feedback and comments.

\section{REFERENCES}

[1] Ferran Altarriba Bertran, Elena Márquez Segura, and Katherine Isbister. 2020. Technology for Situated and Emergent Play: A Bridging Concept and Design Agenda. In Proceedings of the 2020 CHI Conference on Human Factors in Computing Systems (CHI '20), Association for Computing Machinery, New York, NY, USA, 1-14. DOI:https://doi.org/10.1145/3313831.3376859

[2] Ferran Altarriba Bertran, Laia Turmo Vidal, Ella Dagan, Jared Duval, Elena Márquez Segura, and Katherine Isbister. 2020. Chasing Play with Instagram: How Can We Capture Mundane Play Potentials to Inspire Interaction Design? In Extended Abstracts of the 2020 CHI Conference on Human Factors in Computing Systems (CHI EA '20), Association for Computing Machinery, New York, NY, USA, 1-8. DOI:https://doi. org/10.1145/3334480.3382913

[3] Ferran Altarriba Bertran, Elena Márquez Segura, Jared Duval, and Katherine Isbister. 2019. Chasing Play Potentials: Towards an Increasingly Situated and Emergent Approach to Everyday Play Design. Conf. Des. Interact. Syst. (2019). DOI:https://doi.org/10.1145/3322276.3322325

[4] Julia Chan. Top Apps Worldwide for September 2020 by Downloads. Sensor Tower Blog. Retrieved December 9, 2020 from https://sensortower.com/blog/top-apps-worldwide-september2020-by-downloads

[5] Eric Cheng. 2015. Aerial Photography and Videography Using Drones. Peachpit Press.

[6] Honghao Deng, Jiabao Li, Allen Sayegh, Sebastian Birolini, and Stefano Andreani. 2018. Twinkle: A flying lighting companion for urban safety. In Proceedings of the Twelfth International Conference on Tangible, Embedded, and Embodied Interaction, 567-573.

[7] Bill Gaver and John Bowers. 2012. Annotated portfolios. Interactions 19, 4 (July 2012), 40-49. DOI:https://doi.org/10.1145/2212877.2212889

[8] William Gaver. 2002. Designing for homo ludens I3 Mag. 12, (June 2002), 4.

[9] William Gaver. 2012. What should we expect from research through design? Proc. SIGCHI Conf. Hum. Factors Comput. Syst. (May 2012), 937-946. DOI:https://doi. org/10.1145/2207676.2208538

[10] David H. Gobeli and Daniel J. Brown. 1987. Analyzing Product Innovations. Res. Manag. 30, 4 (July 1987), 25-31. DOI:https://doi.org/10.108 $0 / 00345334.1987 .11757048$

[11] C. Hieida, H. Matsuda, S. Kudoh, and T. Suehiro. 2016. Action elements of emotional body expressions for flying robots. In 2016 11th ACM/ IEEE International Conference on Human-Robot Interaction (HRI), 439-440. DOI:https://doi. org/10.1109/HRI.2016.7451795

[12] Natalie Hope McDonald. 2018. Drones on the Upswing. Consumer Technology Association. Retrieved August 25, 2020 from https:/www. cta.tech/Resources/i3-Magazine/i3-Issues/2018/ September-October/Drones-on-the-Upswing

[13] Yunus Karaca, Mustafa Cicek, Ozgur Tatli, Aynur Sahin, Sinan Pasli, Muhammed Fatih Beser, and Suleyman Turedi. 2018. The potential use of unmanned aircraft systems (drones) in mountain search and rescue operations. Am. J. Emerg. Med. 36, 4 (April 2018), 583-588. DOI:https:// doi.org/10.1016/j.ajem.2017.09.025

[14] Matjaž Kljun, Klen Čopič Pucihar, Mark Lochrie, and Paul Egglestone. 2015. StreetGamez: A Moving Projector Platform for Projected Street Games. In Proceedings of the 2015 Annual Symposium on Computer-Human Interaction in Play (CHI PLAY '15), Association for Computing Machinery, New York, NY, USA, 589-594. DOI:https://doi.org/10.1145/2793107.2810305

[15] Joseph La Delfa, Mehmet Aydin Baytaş, Emma Luke, Ben Koder, and Florian "Floyd" Mueller. 2020. Designing Drone Chi: Unpacking the Thinking and Making of Somaesthetic HumanDrone Interaction. In Proceedings of the 2020 ACM Designing Interactive Systems Conference (DIS '20), Association for Computing Machinery, New York, NY, USA, 575-586. DOI:https://doi. org/10.1145/3357236.3395589

[16] Rafat Mahmood and Michael Jetter. 2019. Military Intervention Via Drone Strikes. Social Science Research Network, Rochester, NY. Retrieved August 25, 2020 from https://papers.ssrn. com/abstract $=3390307$ 
[17] Kei Nitta, Keita Higuchi, and Jun Rekimoto. 2014. HoverBall: augmented sports with a flying ball. In Proceedings of the 5th Augmented Human International Conference $(\mathrm{AH}$ '14), Association for Computing Machinery, New York, NY, USA, 1-4. DOI:https://doi. org $/ 10.1145 / 2582051.2582064$

[18] Donald A. Norman and Roberto Verganti. 2013. Incremental and Radical Innovation: Design Research vs. Technology and Meaning Change. Des. Issues 30, 1 (December 2013), 78-96. DOI:https://doi.org/10.1162/DESI_a_00250

[19] Jaime Perez. 2020. Innovating to Fight COVID-19: Four Ways Drones are Contributing. DJI Enterprise. Retrieved August 25, 2020 from https://enterprise.dji.com/news/detail/fight-covid19-with-drones

[20] Andrzej Romanowski, Sven Mayer, Lars Lischke,
Krzysztof Grudzień, Tomasz Jaworski, Izabela Perenc, Przemysław Kucharski, Mohammad Obaid, Tomasz Kosizski, and Paweł W. Wozniak. 2017. Towards Supporting Remote Cheering during Running Races with Drone Technology. In Proceedings of the 2017 CHI Conference Extended Abstracts on Human Factors in Computing Systems (CHI EA '17), Association for Computing Machinery, New York, NY, USA, 2867-2874. DOI:https://doi.org/10.1145/3027063.3053218

[21] Amarjot Singh, Devendra Patil, and S. N. Omkar. 2018. Eye in the Sky: Real-Time Drone Surveillance System (DSS) for Violent Individuals Identification Using ScatterNet Hybrid Deep Learning Network. 1629-1637. Retrieved August 25, 2020 from https://openaccess.thecvf.com/ content_cvpr_2018_workshops/w33/html/Singh Eye in the_CVPR_2018_paper.html
[22] Donna Spencer. 2004. Card sorting: a definitive guide. Retrieved August 25, 2020 from http:// www.iimagineservicedesign.com/wp-content/ uploads/2015/07/Card-sorting-a-definitive-guide$\% \mathrm{C} 2 \% \mathrm{AB}-\mathrm{Boxes}$-and-Arrows.pdf

[23] Frank H. Tainter. 2002. What Does Mistletoe Have To Do With Christmas? APSnet Features. Retrieved August 25, 2020 from https://www. apsnet.org/edcenter/apsnetfeatures/pages/mistletoe.aspx

[24] 2016 China's Drone Industry Report. iResearch Global. Retrieved August 25, 2020 from http:// www.iresearchchina.com/content/details8_22801. html

[25] Crazy 8's. Design Sprints, Google. Retrieved August 25, 2020 from https://designsprintkit. withgoogle.com/methodology/phase3-sketch/ crazy-eights 